\title{
Monitorización de prácticas de disenso y coproducción de conocimiento: el mapa de los conflictos del agua de Andalucía (España)
}

\section{Leandro del Moral* | Belén Pedregal** \\ Cesare Laconi* | Antonio Figueroa** \\ María Mancilla*** | Violeta Cabello***}

Resumen. Primero se constata la generalizada falta de operatividad de las experiencias de gobernanza participativa realmente existentes. Dada esta realidad, se defiende la importancia de monitorizar y aprender de los conflictos, de los disensos, entendidos como «indicadores vivientes» de las realidades que la academia, la política y la sociedad en su conjunto deben abordar con urgencia. Con base en esa reflexión, se plantea la hipótesis de que la coproducción de conocimiento facilitada por el enfoque de cartografía participativa puede ser una buena herramienta para el empoderamiento social y la mejora de los procesos de decisión. El artículo presenta también algunos aspectos de la experiencia del mapa de los conflictos del agua en Andalucía que, incardinado orgánicamente en organizaciones y movilizaciones ciudadanas preexistentes, aspira a aportar resultados positivos, tanto de carácter instrumental como social, a una determinada escala, la regional, y en una materia política específica, el agua.

Palabras clave: coproducción de conocimiento, cartografía participativa, conflictos ambientales, justicia hídrica, Andalucía.

* España. Departamento de Geografía Humana, Universidad de Sevilla.

** España. Red Andaluza de la Nueva Cultura del Agua, España.

*** España. Stockholm Resilience Centre, Stockholm University, Suecia.

**** España. Instituto de Ciencia y Tecnología Ambiental, Universidad Autónoma de Barcelona. 


\section{Tracking design practices and the co-production of knowledge: Mapping water conflicts in Andalucía (Spain)}

Abstract. We begin by documenting the overall operational failure of the experiences of participative governance that are currently in place. In that context, we can see the importance of observing and learning from the conflicts, understood as «living indicators» of the realities that academia, politics and society together should urgently engage. With that in mind, a hypothesis is offered: that the co-production of knowledge facilitated by the focus on participative cartography can be a useful tool for social empowerment and improvement in decision making processes. The article also reveals some aspects of the mapping experience of water-related conflicts in Andalucía which, developing organically within existing civil organizations and movements, seek to deliver positive results — as much instrumental as social_ at a given regional scale, and in one policy area particularly: water.

Keywords: co-creation of knowledge, participative cartography, environmental conflicts, water justice. 


\section{Expectativas y resultados de la gobernanza participativa y el «gobierno más allá del Estado»}

Ya desde hace un par de décadas se viene discutiendo sobre la idea de gobierno abierto, definido como un modelo de interacción sociopolítica basado en la transparencia, la rendición de cuentas, la participación y la colaboración entre los agentes político-sociales. Una estrategia favorable a una manera de gobernar dialogante, con mayor equilibrio entre el poder de los gobiernos y el de los gobernados, que le reconoce a la ciudadanía un papel corresponsable en la toma de decisiones (OECD, 2009). Sin embargo, también desde hace tiempo diversos estudios han revisado con una perspectiva crítica la efectividad real de los enfoques de la gobernanza participativa en los procesos de decisión, tanto en términos procedimentales como sustantivos (Del Moral, 2017). En relación con los requerimientos de información y participación pública, estos estudios han mostrado que, efectivamente, en comparación con etapas anteriores, se ha producido un aumento sustancial de la información compartida por las administraciones gracias a las tecnologías de la información (Hernández y De Stefano, 2011; De Stefano et al., 2012; Hernández et al., 2015). Si bien el aumento de la información disponible y los requisitos legales de participación pública debieran haber implicado una apertura de los procesos de decisión a todos los actores, los estudios realizados muestran que los agentes con más poder, en cada contexto concreto de la política de que se trate, siguen decidiendo prácticamente en solitario los aspectos fundamentales objeto de debate (Parés et al., 2015, Godinez-Madrigal et al., 2019). Incluso en los procesos deliberativos como los que se animan en contextos de gobernanza avanzada, 
las decisiones finales quedan generalmente fuera de la esfera pública y responden a dinámicas de poder que no son explícitas. Algunos autores sostienen que los enfoques de gestión «gobierno más allá del Estado» (governance beyond the state), orientados a alcanzar «consensos» y al «establecimiento racional de prioridades», fuerzan a los actores a colaborar dentro de un marco establecido que no puede ser cuestionado o alterado y que condiciona profundamente los términos del debate y, por consiguiente, los resultados de las políticas (Peck y Tickell, 2002; Swyngedouw, 2011). Estos y otros autores plantean que, al promoverse enfoques de gobernabilidad por parte de las administraciones, los activistas son «desactivados» y obligados a reemplazar los actos de contestación y protesta política por procesos de colaboración con el Estado (Molle, 2009; Parés, 2010).

Concretamente en la política del agua de la Unión Europea, la actual normativa definida por la Directiva Marco del Agua (Directiva 2000/60/ CE, DMA) exige procesos muy ambiciosos de participación pública activa, en coherencia con el Convenio de Aarhus de 19981 (Hernández et al., 2014). No obstante, la entidad real de estos procesos, así como sus resultados operativos, distan mucho de los planteamientos y objetivos establecidos en este marco normativo, como han puesto de relieve los resultados de diversos estudios (Espluga et al., 2011; Ballester y Parés, 2013). El equilibrio de poder sigue favoreciendo a los grandes usuarios de agua, dejando de lado los intereses de los grupos escasamente representados. Estos grupos minoritarios (intereses patrimoniales y ambientales locales, usuarios recreativos e

\footnotetext{
${ }^{1}$ La Comisión Económica de Naciones Unidas para Europa adoptó en Aarhus (Dinamarca) el 25 de junio de 1998 la Convención sobre el acceso a la información, la participación del público en la toma de decisiones y el acceso a la justicia en temas ambientales, conocido como Convenio de Aarhus.
} 
identitarios del agua o poblaciones rurales afectadas por grandes obras de infraestructura) suelen recurrir a acciones de protesta política de mayor o menor éxito (Tàbara e Ilhan, 2008; Arrojo et al., 2010; Subirats, 2011; Poma, 2019). El modelo de gobernanza ofrecido por la DMA fue en general bien acogido por tales actores como una oportunidad para participar en las decisiones sobre el agua en condiciones de igualdad. Sin embargo, tampoco en este caso se han podido evitar esos fenómenos de «desactivación» y sustitución de la protesta política por los mencionados procesos de colaboración, en un marco de continuidad de los mecanismos de decisión controlados por los agentes clave de la comunidad tradicional de política del agua (Parés et al., 2015; Swyngedouw, 2019).

A partir de dicha constatación fundamental, cobra una gran importancia identificar y facilitar la expresión de la conflictividad que está en el núcleo de las interrelaciones de la sociedad y los recursos naturales. Como escribe Maria Kaika, de cara a apoyar procesos de toma de decisión mejor informados, es una responsabilidad académica, política y socioambiental empezar a preguntarse una serie de cuestiones diferentes a las convencionales: «Monitorizar, documentar y almacenar sistemáticamente prácticas y métodos guiados por el disenso, se ha convertido una materia de emergencia política» (2018:99). Si nos tomáramos en serio esas prácticas, podríamos ir más allá de conjuntos de indicadores desfasados y avanzar hacia un marco de señales y criterios orientado a la igualdad socioambiental global. Es imperioso teorizar y comprobar empíricamente los procesos mediante los cuales se producen determinadas configuraciones que generan condiciones sociohidrológicas inequitativas (Swyngedouw, 2019:51).

Al descender a una concreción operativa de estos planteamientos, en el caso de los conflictos territoriales se han puesto de manifiesto en los últimos 
años las potencialidades de la cartografía social para identificar y caracterizar tales prácticas y métodos guiados por el disenso y generadores de conflicto. En ese sentido, existen experiencias de gran interés, como el Atlas de la Justicia Ambiental (Temper y Martínez, 2015), que integran los fundamentos de la cartografía social, a la que luego nos referiremos, y el paradigma de la justicia ambiental, basado en el reconocimiento de la desigualdad en la distribución espacial y social de los efectos de las políticas ambientales (Martínez y O’Connor, 1999; Arriaga y Pardo, 2011). Cabe resaltar que ni éste ni otros trabajos similares, que otros autores (Herrero, 2015) y nosotros mismos hemos analizado en otro lugar (Laconi et al., 2018), recogen con suficiente detalle los conflictos a escala local y regional, así como los referidos específicamente a una sola temática, en nuestro caso, el agua. Y estos, como explicamos a continuación, son elementos importantes de nuestra propuesta metodológica y de nuestros objetivos tecnopolíticos.

\section{Coproducción de conocimiento crítico} a través de cartografía participativa

Sobre la base de estas ideas, el artículo analiza la experiencia de un proceso de coproducción de conocimiento acerca de los conflictos ambientales en una arena de política concreta (el agua) y a una escala regional (Andalucía). ${ }^{2}$ La experiencia forma parte de la construcción y funcionamiento del mapa de los conflictos del agua de Andalucía (Mapa-RedNCA, en adelante) que tras dos años de trabajo se encuentra operativo desde junio de

${ }^{2}$ El caso de estudio se sitúa en Andalucía, una región del sur de España, con una extensión de 87 mil $268 \mathrm{~km}^{2}$ y 8 millones 414 mil 240 habitantes en 2019. 
2019 y ya está siendo analizado desde diferentes perspectivas (Pedregal et al., 2018; Laconi et al., 2019). En este caso nos interesa fundamentalmente la relación que la experiencia tiene con los debates teórico-prácticos en cuanto a coproducción de conocimiento.

Entendemos por coproducción la implicación activa de ciudadanos e investigadores en la generación compartida de información, conocimiento, ideas y experiencias para transformar realidades y construir proyectos (Ruiz, 2020). Dicho enfoque supone el reconocimiento, por parte de los expertos y los responsables de las decisiones, del papel crucial que tienen el conocimiento, la experiencia y las preferencias locales para desarrollar con éxito estrategias y prácticas innovadoras. Implica también una concepción teorético-práctica de la construcción de conocimiento. El concepto no es en absoluto unívoco, sino que, como tantos otros (gobernanza, sostenibilidad, resiliencia, transdisciplinariedad...) es intrínsecamente polisémico y connotativo, y por ello está sometido a múltiples interpretaciones (Van der Hel, 2016; Subirats, 2016; Sorrentino et al., 2018; Norström et al., 2020).

En su dimensión más teórica, coproducción tiene que ver con las relaciones entre modalidades de conocimiento científicas y no científicas, un aspecto al que la literatura denomina de diversas formas, desde «ciencia postnormal», una de las acepciones de la «transdisciplinariedad» propuesta en el debate europeo (Funtowicz y Ravetz, 2000), hasta «diálogo de saberes» (Leff, 2003) y «ecología de saberes» (De Sousa, 2007), entre otras (Castro, 2019). En un terreno más concreto, en su análisis de los procesos de participación y coproducción de conocimiento en el campo de la gobernanza de agua, Lepenies et al. (2018) explican que existen dos grandes tradiciones: coproducción en la perspectiva de los servicios públicos y 
coproducción en la perspectiva de las ciencias de la sostenibilidad, y cada perspectiva conlleva una intención determinada, que puede denominarse técnico-instrumental y de empoderamiento social, respectivamente, con implicaciones políticas diferenciadas. En esa misma línea, en la literatura sobre cambio climático, se entiende coproducción de dos maneras diferentes, aunque conectadas entre sí (Kerkhoff y Lebel, 2015). Por un lado, como una noción instrumental, interesada en la participación social para enriquecer las bases científicas de los procesos de decisión; por otro, como una práctica que trata de desvelar la naturaleza de las desiguales relaciones de poder que rodean la investigación y la política de cambio climático a diferentes escalas (Ruiz, 2020). Pese a que la experiencia del Mapa-RedNCA contiene elementos de ambos enfoques (Pedregal et al., 2020), en este artículo nos centramos en su dimensión de herramienta para el empoderamiento social, un enfoque de largo recorrido que conecta con la Pedagogía del oprimido, de Freire (1970), orientada a que los desfavorecidos identifiquen y analicen los riesgos que afrontan a fin de convertirse en protagonistas de la mejora de su realidad; o con el concepto de «conocimiento activista» de Escobar (2016), que sostiene que los movimientos sociales, por medio de la protesta y la contestación cultural, son espacios importantes de producción de conocimiento y no sólo de activación de políticas.

Desde la perspectiva de las herramientas cartográficas utilizadas, la experiencia del Mapa-RedNCA se apoya en la distinción entre cartografía participativa, entendida como aquellos procesos cartográficos que implican dinámicas participativas cara a cara, en procesos presenciales, abiertos e inclusivos; y proyectos de cartografía colaborativa que se centran fundamentalmente en la recopilación y difusión de datos geográficos aportados de manera voluntaria por individuos que funcionan como «sensores voluntarios» 
(Goodchild, 2007). En ese sentido, siguiendo a Liu et al. (2018), distinguimos entre procesos cartográficos participativos de base comunitaria, y aquellas prácticas de cartografía colaborativa apoyadas fundamentalmente en plataformas web diseñadas para crear y compartir información de base geográfica por parte de voluntarios.

Como buena parte de las experiencias de cartografía participativa (Risler y Ares, 2015; Ortega, 2011; Corbett, Cochrane y Gill, 2016), el Mapa-RedNCA tiene el objetivo de generar espacios de intercambio colectivo de narrativas que pongan en cuestión la lógica de los discursos hegemónicos, permitiendo que informaciones y conocimientos vernáculos, escasamente visibilizados por los discursos convencionales, se abran espacio entre las representaciones dominantes. Una de las principales características de nuestro caso es que el proyecto de construcción del mapa y su propio resultado como visor cartográfico no se superponen como una creación exógena al movimiento social, aunque sea de una forma dialogante, sino que se integran orgánicamente en él. Desde los comienzos de su proceso de construcción el proyecto se ha incardinado en prácticas de organización y movilización social preexistentes, que han facilitado la difícil tarea de la coproducción. En efecto, el Mapa-RedNCA entrelaza el proyecto de investigación con la estructura organizativa y comunicativa de la Red Andaluza de la Nueva Cultura del Agua (Red-ANCA), una coordinadora de ámbito regional, que funciona desde 2004 y que aborda los problemas relativos a los ecosistemas y patrimonios del agua con vocación integradora, a la vez crítica, reivindicativa, propositiva y dialogante, con independencia de estructuras estatales y administrativas (incluidos partidos). Su funcionamiento no pretende ir más allá de lo que demanden las entidades y colectivos sociales que la integran, extendidos por toda la región. Trabaja 
tanto en red como presencialmente, dando a ambos modos de consulta y toma de decisiones similar legitimidad (Red Andaluza de la Nueva Cultura del Agua, 2017). Un aspecto de la materialización de esta hibridación, a la que aspiran las experiencias de coproducción de conocimiento, es la incorporación del mapa en la página web de la Red-ANCA, a la que a su vez el proyecto aporta contenido y desarrollo (Red Andaluza de la Nueva Cultura del Agua, s/f). Dicha integración, que constituye una de las principales originalidades y potencialidades de esta experiencia, aumenta las posibilidades de imbricación en el tejido social y de mantenimiento en el tiempo del mapa, dos de las grandes dificultades de ese tipo de proyectos.

Dicha estrategia está permitiendo la incorporación y reconocimiento del mapa por los colectivos, lo que aumenta su operatividad y sus posibilidades de mantenimiento en el tiempo, más allá de la vida administrativa del proyecto científico-académico, que también es parte fundamental del proceso. Las consecuencias de la inserción también se producen en términos de difusión de la experiencia en diferentes medios crítico-alternativos de significativo impacto social, como las revistas El Salto y El Ecologista, editadas en Madrid, que en sus números correspondientes a abril 2019 y de invierno 2019-2020, respectivamente publican sendos reportajes dedicados al Mapa-RedNCA (Otero, 2 de mayo de 2019; Ecologistas en Acción, 15 de diciembre de 2019). También la organización ONGAWA, Ingeniería para el Desarrollo Humano recoge este proyecto como una destacada experiencia de iniciativas sociales de adaptación al cambio climático. ${ }^{3}$ Mientras que algunos diversos proyectos científicos, como ResCities coordinado por la Universitat Oberta de Catalunya, se hacen eco también de la experiencia (IN3 Blog, 25 de noviembre de 2019).

${ }^{3}$ Véase https://ongawa.org/ 


\section{La construcción de la herramienta: el Mapa-RedNCA}

Cualquier proyecto de cartografía participativa se enfrenta con la tarea de diseñar una herramienta capaz de facilitar la incorporación de información de manera autónoma por parte de los ciudadanos. Tal objetivo requiere un especial esfuerzo en el diseño de la herramienta desde dos puntos de vista: en primer lugar, desde un punto de vista tecnológico, con objeto de facilitar el manejo autónomo de la herramienta a usuarios no expertos; en segundo lugar, desde un punto de vista ontológico y semántico, con el propósito de determinar inequívocamente qué información ha de ser incorporada y en qué términos, mediante la construcción de un lenguaje común (técnico, experto, coloquial).

Ambas condiciones fueron abordadas paralelamente desde los inicios del proyecto, estando las primeras fases de construcción del visor cartográfico dedicadas al análisis de antecedentes, con particular atención a los modelos de coproducción, gestión de la información y publicación de la información compartida. Al mismo tiempo, se analizaron las distintas opciones de software, sus funcionalidades y prestaciones. Teniendo en cuenta las limitaciones de tiempo y dotación presupuestaria, y dado el objetivo de fomentar el carácter abierto en todas las fases del proyecto, se optó por el software libre Ushahidi, diseñado expresamente para el activismo social.

Paralelamente, se llevó a cabo la definición de los contenidos del mapa: qué cartografiar y qué información asociada resultaría de interés. Estas tareas se desarrollaron a lo largo de las etapas y métodos siguientes:

a) El análisis de antecedentes: por un lado, mediante la revisión de proyectos de cartografías colaborativas en un sentido amplio; por otro lado, al definir y categorizar los conceptos en el mapa, que tuvieron como 
punto de referencia inicial los documentos técnicos de la planificación hidrológica.

b) La discusión entre los miembros del equipo, formado por investigadores de distintas trayectorias y disciplinas: antropología, geografía humana, ciencias políticas, ciencias ambientales e informática. Este proceso desembocó en una primera propuesta de formato para documentar los conflictos, así como una primera versión de la interfaz del visor cartográfico y su modelo de gestión: registro libre y publicación supervisada. A partir de aquí se puso en marcha el proceso de coproducción del mapa mediante dos acciones principales:

1. Entrevistas a académicos y activistas. En concreto, se realizaron 31 entrevistas en profundidad, de aproximadamente 60-90 minutos de duración, a activistas de todas las provincias andaluzas, seleccionados mediante el método bola de nieve a partir de los contactos de la Red-ANCA.

2. Tres talleres orientados a codiseñar los contenidos de la herramienta y otros tres dedicados a difundir el proyecto y promover la consulta y contribución de información sobre la herramienta ya consolidada. Los talleres se celebraron en su mayoría en el contexto de asambleas anuales convocadas por los movimientos ciudadanos (ecologistas, defensores del patrimonio territorial y activistas del derecho humano al agua) o dentro del marco de iniciativas de educación ambiental promovidas por la administración regional. Sólo uno de ellos fue específicamente organizado por el equipo de trabajo, en colaboración con el Instituto de Estadística y Cartografía de Andalucía con objeto de incorporar la visión de otros perfiles más técnicos-administrativos al codiseño de la herramienta.

Una vez cerrado el proceso de diseño del visor cartográfico y la ficha de información, la siguiente fase del proyecto estuvo dedicada a distintas 
campañas de difusión con un doble objetivo: por un lado, fomentar las contribuciones autónomas a la herramienta y, por otro, promover la sensibilización social de los conflictos representados en el mapa. En específico, se realizaron las siguientes actividades: a) Realización de un video de presentación del proyecto y dos videos tutoriales sobre la herramienta, (enero-febrero de 2019) publicados en Youtube, enlazados a la página web del mapa y difundidos a través de las redes sociales de la Red-ANCA. b) Difusión de los contenidos del mapa mediante dos publicaciones mensuales de dos conflictos concretos (febrero-agosto de 2019) a través de la cuenta de la Red-ANCA en Facebook y Twitter. Por medio de tales acciones, el diseño tanto de la interfaz del visor cartográfico como de la información que contiene ha sido negociado, discutido y acordado entre los miembros del equipo y los actores involucrados.

En el mismo marco de imbricación dinámica entre investigadores y activistas, se planteó también la discusión más amplia acerca de modelos de gestión: ¿moderación por experto o registro abierto? ¿Podría la propia comunidad de usuarios actuar como moderadora? ¿Qué modelo garantiza mejor la sostenibilidad en el tiempo a la vez que se mantiene la calidad de la información? Si bien la intención original del equipo fue crear una herramienta autónoma, sin necesidad de supervisión, que pudiera integrarse y apoyar la acción de la organización social en la que se inscribe, el análisis de antecedentes (Laconi et al., 2018) y las opiniones recabadas durante las propias entrevistas y talleres nos hicieron cambiar de opinión. Efectivamente, la mayoría de los entrevistados opina que tanto el registro para añadir información al mapa como la creación y publicación de los informes deberían ser supervisados, con la finalidad de garantizar la calidad de la información introducida y de esa forma su credibilidad. 
Una de las cuestiones que ha suscitado mayor dificultad en la construcción de la herramienta ha sido la tipificación del origen del conflicto. Tras un largo proceso de debate en el seno del equipo investigador, compartido y contrastado en los talleres con agentes sociales mencionados, la ficha se configuró de manera que integra toda la tipología de presiones incluidas por la Directiva Marco del Agua (DMA) europea, del modo en que la Instrucción de Planificación Hidrológica (Orden ARM/2656/2008) la concreta y desarrolla en el marco normativo español. El objetivo es que la información recogida sea lo más formalizable posible en los términos usados por los procesos de gestión y planificación formales derivados de la aplicación de la DMA. Pero desde un principio el equipo investigador entendió que la batería de conflictos que se pretendía cartografiar, integrando en el proceso a los protagonistas de esas experiencias, desbordaría el marco de esas ya amplias categorías.

Efectivamente, en el marco conceptual de la DMA no hay espacio para algunas demandas sociales como la defensa de los patrimonios y paisajes del agua, la garantía del derecho humano al agua o la defensa de usos no económicos de agua, ya sean recreativos o culturales. Por esta razón, se incorporaron al mapa otras categorías de conflicto, que tienen en cuenta, por una parte, la presencia de los paisajes y de los patrimonios culturales del agua, fundamentales en el entendimiento social de la naturaleza y, más en concreto, de los ecosistemas acuáticos; y por otro, la importancia de la participación ciudadana en los procesos de defensa de la gestión pública de los servicios urbanos de agua o de su remunicipalización en caso de situaciones de privatización. De esta hibridación de perspectivas surgió una estructuración compleja, ambiciosa, demandante de atención y esfuerzo por parte de los colectivos cooperadores, pero sin duda rica y con considerable 
potencial de aportación de información precisa y procesable con diferentes herramientas que puede acabar produciendo conocimiento.

\section{Resultados: caracterización de la conflictividad por el agua en Andalucía}

Después de dos años de trabajo de construcción, el Mapa-RedNCA está operativo desde mediados de 2019 y actualmente incluye alrededor de 65 casos (figura 1). El mapa representa la localización exacta del conflicto con una ficha asociada de información que contiene la descripción textual del mismo por parte de los actores involucrados, además de las siguientes secciones y campos:

Tipología del conflicto: en este apartado del mapa los informantes pueden elegir mediante selección múltiple los tipos y subtipos de conflicto (7 tipos y 25 subtipos).

Ámbito: compuesto por las categorías natural, rural, periurbano y urbano.

Escala del conflicto: que puede definirse en los niveles local, comarcal, regional, nacional e internacional.

Masa de agua afectada: superficial o subterránea.

Los actores involucrados: apartado donde se describe mediante texto abierto qué organizaciones sociales, actores institucionales, entidades y empresas privadas han estado involucradas en las dinámicas del conflicto.

Conflicto y movilización: sección en la que el usuario tiene la posibilidad de proporcionar datos sobre la cronología del conflicto, la intensidad (en su momento álgido), la forma de movilización, el nivel de participación 
de la ciudadanía en los procesos institucionales, el nivel de información aportada por las instituciones, la disponibilidad y el acceso a la información por parte de la ciudadanía.

Impactos: divididos en las tres categorías a) medioambientales, b) socioeconómicos y c) sobre la salud.

Resultados: sección donde se pueden especificar detalles acerca del estado del proyecto asociado al conflicto (si existe) que ha generado actuaciones sobre la masa de agua, las alternativas al proyecto propuestas por la sociedad civil y el éxito (o fracaso) de la movilización.

Recursos y materiales: documentación escrita y gráfica complementaria.

Figura 1

Mapa participativo de los conflictos del agua de Andalucía (España)

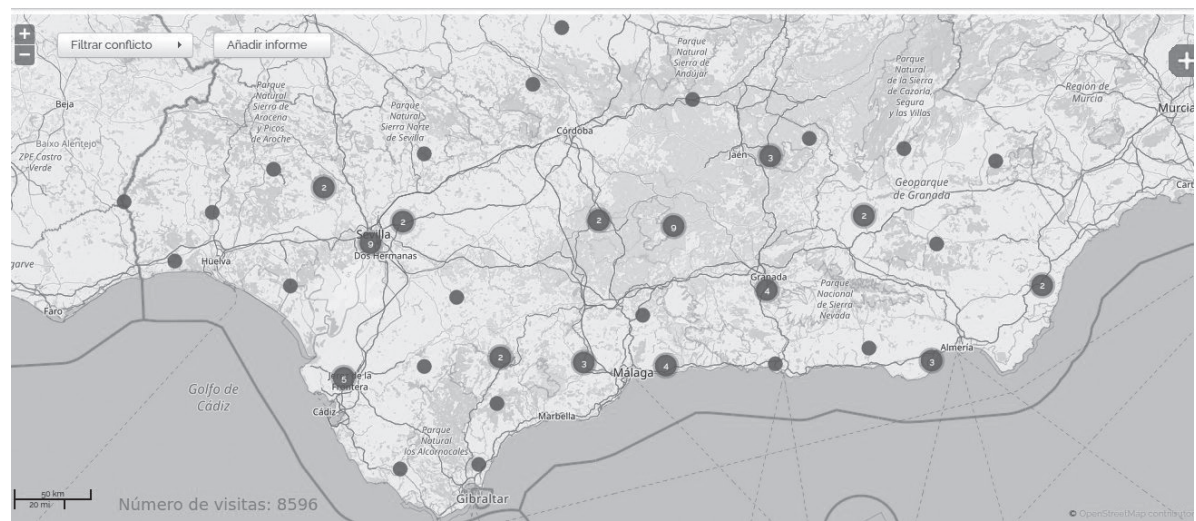

Nota: los puntos pequeños indican la localización de un conflicto. Los puntos mayores identifican la localización de dos o más conflictos, que al hacer zoom sobre el mapa se desagregan y localizan con mayor precisión.

Fuente: Red Andaluza Nueva Cultura del Agua (s/f). 
Los conflictos registrados se distribuyen por Andalucía de manera sensiblemente homogénea, pues están presentes en la totalidad de las grandes unidades territoriales y cuencas hidrográficas. El protagonismo de la protesta corresponde a movimientos sociales autoorganizados, por lo general sin ningún tipo de articulación institucional formal: las formas más básicas de organización (las «plataformas» y las «coordinadoras») aparecen en el mapa en una posición elevada de protagonismo, tras la categoría «vecinos». Esta es una información sumamente relevante. A través de esas estructuras básicas, que con frecuencia ni siquiera se constituyen en «asociaciones culturales sin ánimo de lucro», que es la más sencilla de las fórmulas de legalización, los diferentes grupos constituidos en tanto que «afectados» se transforman en actores políticos que plantean sus demandas en términos de justicia espacial e intergeneracional.

Destaca también la importancia de las organizaciones ecologistas, especialmente y con diferencia, la organización Ecologistas en Acción, que con diversas concreciones locales aparece extendida por gran parte del territorio andaluz, con una estructura federal de gran autonomía y usualmente con fuerte identidad local. A lo largo de los últimos años esta organización ha incrementado su capacidad de intervención, con una considerable acumulación de experiencia y cualificación técnica por parte de un grupo significativo de activistas.

Es llamativa también la escasa presencia en la movilización de entidades del mundo del trabajo y la economía (sindicatos y asociaciones empresariales), que, por el contrario, frecuentemente se sitúan en el bloque social que defiende los proyectos o los procesos de transformación que generan el conflicto. Destaca, además, la escasa presencia de la administración municipal entre los actores movilizados; no es raro que los ayuntamientos, 
como en el caso anterior, apoyen las agresiones que generan el conflicto, aunque con situaciones y dinámicas locales muy contrastadas.

Dada la naturaleza en buena parte espontánea del movimiento, por lo común con una organización frágil y con débiles o nulos procesos institucionales de gobernanza, el conflicto es de carácter reactivo: se inicia, en la mayor parte de los casos, cuando una parte mayor o menor de la población local percibe el inicio del proyecto o incluso tras empezar a sufrir sus perjuicios, es decir, como reacción a los mismos. Sobresale, sin embargo, el aumento de la capacidad discursiva, organizativa y de acción jurídico-institucional del asociacionismo ambiental que da soporte a las movilizaciones.

Por lo que se refiere a los aspectos relacionados con el acceso a la información y participación en procesos institucionales, las informaciones recogidas ponen de manifiesto importantes déficits al respecto. Los afectados expresan quejas en materia de información, incluyendo desconocimiento, falta de disponibilidad o accesibilidad, existencia de ocultación o trabas a su utilización. Los afectados advierten que la información, aun estando disponible, en la mayoría de los casos no se puede reutilizar, lo que contraviene la vigente normativa en materia de acceso a la información ambiental. Los datos recogidos muestran también otra constante clave en el desarrollo de los conflictos del agua: la ciudadanía y los activistas entrevistados señalan y denuncian reiteradamente la inexistencia o debilidad de procesos de participación pública, lo que contrasta con las ambiciosas exigencias en este aspecto de la normativa vigente en materia de medio ambiente y, específicamente, de agua.

Como una de las conclusiones de más calado, la experiencia de elaboración del Mapa-RedNCA está mostrando que la relación de la sociedad 
andaluza con el sistema hidrológico se basa en buena parte en la interrelación histórica, cultural, emocional e identitaria entre las comunidades locales y el agua, es decir en la relación con los paisajes del agua, por más que esa expresión no aparezca hasta momentos recientes en los discursos con los que se construyen estas experiencias (Laconi et al., 2019). En ese sentido el análisis de los resultados del mapa viene a confirmar la importancia del concepto de paisajes del agua, waterscapes en la bibliografía internacional (Budds e Hinojosa, 2012). La incorporación de categorías socioculturales, resultado del proceso participativo, ha permitido expresar la dimensión y vivencia paisajística y patrimonial de un porcentaje muy considerable de los conflictos cartografiados. La destacada presencia de la dimensión sociocultural, sin embargo, no excluye en absoluto la raíz físico-natural, ecológica, de la conflictividad, tanto en aguas superficiales como en subterráneas.

Por su parte, las entrevistas semiestructuradas a significados activistas (recogidas en formato Google Forms), muchos de ellos representantes o portavoces de los colectivos de afectados, han aportado información sobre otros aspectos, como la propia valoración del mapa por parte de los activistas. Casi la totalidad de los entrevistados opina que el mapa puede ser de gran utilidad para su actividad (96 por ciento). En la mayor parte de los casos, esta utilidad se expresa en términos, por una parte, de producción de información sobre conflictos que en ocasiones son poco conocidos más allá de la escala local; y por otra, de generación de interrelaciones entre diferentes experiencias, es decir, de formación de redes. Se confirma de ese modo la capacidad de la herramienta y del propio proceso implementado para generar procesos de empoderamiento al tiempo que el producto resultante supone una base de datos de referencia «alimentada desde el territorio» en palabras de los propios activistas, lo que a su vez apunta hacia 
la utilidad de monitorización de las «prácticas de disenso» (Kaika, 2018) que señalábamos anteriormente.

No obstante, por lo que respecta a la capacidad de influenciar los procesos de toma de decisión, que también está en el ADN de la experiencia (Pedregal et al., 2020), las expectativas no son tan altas. Al responder a la pregunta ¿en qué sentido puede el mapa ayudar al colectivo que representas?, todos los informantes consideran que la herramienta es útil para visibilizar conflictos, compartir información y crear redes de colaboración, pero sólo 74 por ciento ( 42 informantes) considera que podría contribuir a mejorar la gestión del agua en Andalucía. Esta última constatación es relevante, en la medida en que muestra que la herramienta no es suficiente para resolver uno de los grandes retos planteados al principio: por un lado, la fractura entre las altas exigencias de participación pública que el marco normativo establece, en especial en el caso de la gestión del agua, y que la sociedad demanda y, por otro lado, los escasos resultados prácticos que los procesos de participación cosechan en la realidad. Queda por ver en qué medida la continuidad, extensión y afianzamiento en el tejido social e institucional de la herramienta puede producir en el futuro algunos resultados tangibles como instrumento de mejora de la gobernanza.

\section{Conclusiones}

El marco normativo e institucional, en particular en el terreno de la política ambiental, exige abordar la complejidad, las profundas incertidumbres y los grandes intereses en juego en los procesos de decisión mediante la participación de todos los agentes sociales legítimos, apoyado en la información y 
el conocimiento más completo y riguroso posible de los factores de los problemas. En el caso de la política del agua en Europa, la normativa específica, la DMA, establece protocolos y metodologías concretos que pretenden operativizar esta estrategia, a través de la información, consulta y participación activa de las partes interesadas y el público en general en los procesos de planificación de las cuencas hidrográficas. Pero, a pesar del consistente marco institucional, como ya fuera anticipado por el trabajo seminal de Sherry R. Arnstein A ladder of citizen participation, «existe una diferencia crítica entre atravesar rituales vacíos de participación y tener el poder real que se necesita para incidir en los efectos del proceso» (Arnstein, 1969:216).

En este contexto, siguiendo el modelo de otras experiencias, de manera especial la del Environmental Justice Atlas (Temper et al., 2015; 2018), el Mapa-RedNCA aspira a aportar una herramienta para contribuir a la comprensión de situaciones de inequidad en la distribución de recursos territoriales; potenciar el diálogo y el intercambio de experiencias, ideas, datos y estrategias; proporcionar una fuente de información con casos concretos, relevantes y coherentemente sistematizados; sensibilizar a los medios de comunicación y a la propia opinión pública; desarrollar y fortalecer estrategias de articulación de políticas de justicia ambiental a escala regional; $\mathrm{y}$, como base de todo ello, contribuir a procesos de coproducción de conocimiento (Temper et al., 2015).

Como resultado de los procesos de coproducción de los que surge, el Mapa-RedNCA ofrece un documento cartográfico que organiza una información que anteriormente no estaba visibilizada. En la senda de las experiencias anteriores en las que se inspira proporciona material empírico para una agenda de investigación encauzada a entender de qué manera se producen y se interpretan las transformaciones en los sistemas 
socioecológicos, que conllevan desiguales distribuciones de beneficios y perjuicios entre diferentes sectores sociales. Una agenda de investigación dirigida a entender cómo, dónde y con qué resultados se combaten estos efectos por parte de los afectados. En ese sentido, incorporando también el discurso de Maria Kaika, el Mapa-RedNCA aspira a constituir una herramienta útil para monitorizar, documentar y almacenar las prácticas y métodos orientados al disenso (Kaika, 2018).

Una de las características que dota de mayor singularidad a esta experiencia es que desde su origen se imbrica con asociaciones ciudadanas, integradas en la Red Andaluza de la Nueva Cultura del Agua, una organización social de carácter temático, agua, y ámbito regional. La experiencia del Mapa-RedNCA se incardina en procesos de organización y movilización social existentes con anterioridad, a escalas local y regional, y aspira a sostenerse en el tiempo, mantenida en energías sociales autónomas.

Esa ha sido una de las principales preocupaciones del proyecto desde sus inicios, estrechamente relacionada en la práctica con el enfoque de integración anterior: ¿cómo mantener el mapa activo y actualizado más allá del proyecto científico que ha contribuido a él? Una de las formas de materializar esta apuesta ha sido no construir una nueva plataforma web — probablemente de fugaz existencia, como las que caracterizan habitualmente a los proyectos de investigación-, sino situarse, contribuyendo a su mejora y consolidación, en una plataforma preexistente. En ella el equipo científico inserta, impulsa y perfecciona un proyecto y una herramienta de su mismo ámbito escolar — proximidad, carácter comunitario — y temático: el potente factor cohesionador del agua.

De la información disponible hasta el momento hay que decir que, por lo que respecta a la capacidad de influencia real en los procesos de toma de 
decisión, las expectativas son moderadas. Como ya se ha advertido, todos los activistas participantes en el proceso consideran que la herramienta es útil para visibilizar conflictos, compartir información y crear redes de colaboración, pero un sector significativo, en torno a una cuarta parte, no considera que pueda contribuir a mejorar la gestión del agua en Andalucía. Esta constatación es relevante, en la medida en que en ella reconocemos que la herramienta suscita dudas sobre su operatividad para resolver la fractura entre las altas exigencias de participación pública que el marco normativo establece y que además la sociedad demanda, por otra parte, y los escasos resultados prácticos que los procesos de participación cosechan en la realidad.

En relación con las categorías que construyen la tipología de los conflictos es interesante destacar que, como resultado de la metodología aplicada, el mapa incorpora categorías de conflictos vinculados con las dimensiones de patrimonio cultural y de paisaje, pese a que hacerlo así complicaba la claridad de las tipologías derivadas del marco normativo vigente. El desarrollo de la experiencia colaborativa ha venido a demostrar que esta opción fue acertada: la incorporación de categorías socioculturales ha permitido expresar la dimensión y vivencia paisajística y patrimonial de un porcentaje muy considerable de los conflictos cartografiados.

Queda por ver en qué medida la continuidad, extensión y afianzamiento en el tejido social de la herramienta, que es una clave de esta experiencia de cogeneración dinámica e inacabada, pueden ayudar a producir en el futuro algunos resultados tangibles en ese sentido. Como expone Esteban Castro en relación con la Red WaterlatGobacit, la tarea que convoca a la Red Andaluza es la de producir conocimiento dirigido a apoyar los procesos de democratización de la política y la gestión del agua que, a su vez, implica la propia democratización de los procesos de producción 
de conocimiento (Castro, 2019:37). En efecto, se suelen elogiar las prácticas de cartografía digital como instrumentos de democratización y empoderamiento, valorando positivamente las potencialidades de la tecnopolítica, pero la experiencia indica que no es suficiente registrar conflictos y exponer sus argumentos, puesto que nada cambiará a largo plazo en tanto estos conflictos no rebasen la reiteración de disputas locales y denuncias virtuales con escasa repercusión, convirtiéndose en fuerzas sociales y materializándose en la creación de nuevas agendas políticas (Merlinsky, 2016). Este desafío es el más apremiante y requiere un esfuerzo intelectual sostenido, así como la movilización de energías creativas por parte de aquellos que tienen al agua como objeto de investigación.

\section{Reconocimientos}

Este trabajo contó con la financiación del Centro de Estudios Andaluces a través del proyecto «Atlas digital colaborativo de la justicia ambiental en el agua. Contribución a la transparencia y los datos abiertos en las políticas públicas de Andalucía» (PRY125/17). Queremos expresar nuestro agradecimiento a todas las personas que accedieron a ser entrevistadas y que han participado activamente en los talleres desarrollados. Y sobre todo al conjunto de activistas en cuya tarea permanente y cotidiana está basada la consolidación de esta experiencia. 


\section{Referencias}

Arriaga, A. y Pardo, M. (2011). «Justicia ambiental. El estado de la cuestión». Revista Internacional de Sociología, 69(3), pp. 627-648.

Arnstein, S.R. (1969). "A ladder of citizen participation». Journal of the American Institute of Planners, 35(4), pp. 216-224. Recuperado de https://doi. org/10.1080/01944 366908977225

Ballester, A. y Parés, M. (2013). «Democracia deliberativa y política de agua: experiencias de participación en el contexto de la Directiva Marco del Agua en España». En Actas del VIII Congreso Ibérico de Gestión y Planificación del Agua (pp. 178-190). Lisboa: Fundación Calouste Gulbenkian.

Budds, J. e Hinojosa, L. (2012). «Restructuring and rescaling water governance in mining contexts: the co-production of waterscapes in Peru». Water Alternatives, 5(1), pp. 119-137.

Castro, J.E. (2019). «X-disciplinariedades y bifurcaciones en los estudios sobre el agua». En Castro, J.E., Kohan, G., Poma, A. y Ruggerio, C. (eds.), Territorialidades del agua. Conocimiento y acción para construir el futuro que queremos (pp. 18-47). Buenos Aires: Ciccus.

Corbett, J., Cochrane L. y Gill, M. (2016). «Powering up: revisiting participatory GIS and empowerment». The Cartographic Journal, 53(4), pp. 335-340. DOI: https://doi.org/10.1080/00087041.2016.1209624

De Stefano, L., Hernández-Mora, N., López Gunn, E., Willaarts, B., Zorrilla, P. y Llamas, R. (2012). «Public participation and transparency in water management». En De Stefano, L. y Llamas, R. (eds.), Water, agriculture and the environment in Spain: can we square the circle? (pp. 217-225). Balkema: CRC Press.

De Sousa Santos, B. (2007). «Para além do pensamento abissal. Das linhas globais a uma ecologia de saberes». Novos Estudos, 79, pp. 71-94. 


\section{Leandro del Moral | Belén Pedregal | Cesare laconi | Antonio Figueroa}

María Mancilla | Violeta Cabello

Del Moral Ituarte, L. (2017). «Participación: balance de aplicación de la Directiva Marco del Agua y demandas actuales de los agentes sociales». En Embid Irujo, A. (dir.), El futuro de los organismos de cuenca, Cizur Menor (Navarra) (pp. 175-196). Navarra: Thomson Reuters/Aranzadi.

Parlamento Europeo y Consejo de la Unión Europea (23 de octubre de 2000). Directiva 2000/60/C del Parlamento Europeo y del Consejo de 23 de octubre de 2000 por la que se establece un marco comunitario de actución en el ámbito de la política de aguas.

Ecologistas en Acción (15 de diciembre de 2019). «Editorial. La sociedad despierta». Ecologista (102). Recuperado de https://www.ecologistasenaccion. org/132876/editorial-la-sociedad-despierta/

Escobar, A. (2016). «A manera de prólogo». En Merlinsky, G. (coord.), Cartografías del conflicto ambiental en Argentina 2 (pp. 7-11). Buenos Aires: Ciccus.

Freire, P. (2005). Pedagogía del oprimido. México: Siglo XXI.

Espluga, J., Ballester, A., Hernández-Mora, N. y Subirats, J. (2011). «Participación pública e inercia institucional en la gestión del agua en España». Revista de Estudios e Investigaciones Sociales, 134, pp. 3-26.

Figueroa, A., Laconi, C., Del Moral, L. y Pedregal, B. (2019). «Los conflictos del agua en Andalucía». Ecologista (102), pp. 46-49.

Funtowicz, S.O. y Ravetz, J.R. (2000). La ciencia posnormal: ciencia con la gente. Barcelona: Icaria.

Godinez-Madrigal, J., Van Cauwenbergh, N. y Van der Zaag, P. (2019). «Production of competing water knowledge in the face of water crises: revisiting the IWRM success story of the Lerma-Chapala Basin, Mexico». Geoforum, 103, pp. 3-15. DOI: https://doi.org/10.1016/j.geoforum.2019.02.002

Goodchild, M.F. (2007). «Citizens as sensors: the world of volunteered geography». GeoJournal, 69, pp. 211-221. 
Hernández-Mora, N. y De Stefano, L. (eds.) (2011). «Transparencia en la gestión del agua en España». Papeles de Seguridad Hídrica y Alimentaria (4).

Hernández-Mora, N., Cabello, V., De Stefano, L. y Del Moral, L. (2015). «Networked water citizen organizations in Spain: potential for transformation of existing power structures for water management». Water Alternatives, 8(2), pp. 99-124.

Herrero Cabrejas, A. (2015). «Cartografía de los conflictos socioecológicos en el Estado español». Ecología Política, 48, pp. 88-89.

IN3 Blog (25 de noviembre de 2019). «RESCITIES: the limits and potentials of Urban Resilience». Recuperado de https://in3.blogs.uoc.edu/rescities-limits-potentials -urban-resilience/

Kaika, M. (2018). «Between the frog and the eagle: Claiming a «scholarship of presences for the Anthropocene», European Planning Studies, 26 (9).

Laconi, C., Pedregal, B. y Del Moral, L. (2018). «La cartografía colaborativa para un cambio social. Análisis de experiencias». XVIII Congreso Nacional de Tecnologías de la Información Geográfica, Perspectivas Multidisciplinares en la Sociedad del Conocimiento, Valencia.

Laconi, C., Figueroa, A., Pedregal, B., Cabello, V., Mancilla, M. y Del Moral, L. (2019). «La presencia del paisaje en el mapa digital colaborativo de los conflictos del agua en Andalucía». 22 reunión del Taller del Consejo de Europa para la implementación del Convenio del Paisaje del Consejo de Europa y Congreso Internacional «Agua, paisaje y ciudadanía ante el cambio global», 14, 15 y 16 de marzo. Leff, E. (2003). «Racionalidad ambiental y diálogo de saberes: sentidos y senderos de un futuro sustentable». Desenvolvimento e Meio Ambiente (7), pp. 13-40. Lepenies, R., Hüesker, F., Beck, S. y Brugnach, M. (2018). «Discovering the political implications of coproduction in water governance». Water, 10(1475). DOI: 10.3390/w10101475 


\section{Leandro del Moral | Belén Pedregal | Cesare laconi | Antonio Figueroa}

María Mancilla | Violeta Cabello

Liu, Wei, Dugar, Sumit, McCallum, Ian, Thapa, Gaurav, See, Linda, Khadka, Prakash, Budhathoki, Nama, Brown, Sarah, Mechler, Reinhard, Fritz, Steffen y Shakya, Puja (2018). «Integrated participatory and collaborative risk mapping for enhancing disaster resilience». International Journal of Geo-Information, $7(2)$.

Martínez-Alier, J. y O’Connor, M. (1999). «Distributional issues: an overview». En Van den Bergh, J. (ed.), Handbook of environmental and resource economics. Cheltenham: Edward Elgar.

Molle, F. (2009). «Water, politics and river basin governance: repoliticizing approaches to river basin management». Water International, 34(1), pp. 62-70.

Norström, A.V. et al. (2020). «Principles for knowledge co-production in sustainability research». Nature Sustainability. DOI: https://doi.org/10.1038/ s41893-019-0448-2

Otero, A. (2019). «Los conflictos del agua en Andalucía». El Salto de Andalucía (24), pp. 12-16.

Organisation for Economic Co-operation and Development (OECD) (2009). Focus on Citizens: Public Engagement for Better Policy and Services. París: OECD.

Parés, M. (2010). «River basin management planning with participation in Europe: from contested hydro-politics to governance-beyond-the state». European Planning Studies, 19(3), pp. 457-478.

Parés, M., Brugué, Q., Espluga, J., Miralles, J. y Ballester, A. (2015). «Strengths and weaknesses of deliberation on river basin management planning: analysing the Water Framework Directive Implementation in Catalonia (Spain)». Environmental Policy and Governance, 25(2), pp. 97-110.

Peck, J. y Tickell, A. (2002). «Neoliberalizing space». Antipode, 34(3), pp. 380-404. Pedregal, B., Laconi, C., Figueroa, A., Cabello, V., Mancilla, M. y Del Moral, L. (2018). «Mapa digital colaborativo de la justicia ambiental en el agua. 
Planteamientos conceptuales y debates en las primeras etapas de su desarrollo operativo». X Congresso Ibérico de Gestão e Planeamento da Água. Coimbra. Recuperado de http://congresoiberico.org/

Pedregal, B., Laconi, C. y Del Moral, L. (2020). «Promoting environmental justice through integrated mapping approaches: the map of water conflicts in Andalusia (Spain)», International Journal of Geo-Information, 9(2).

Poma, A. (2019). «Resistir para existir. Una propuesta analítica para comprender la dimensión subjetiva de los conflictos contra represas desde la perspectiva de los afectados». En Castro, J.E., Kohan, G., Poma, A. y Ruggerio, C. (eds.), Territorialidades del agua. Conocimiento y acción para construir el futuro que queremos (pp. 93-115). Buenos Aires: WaterlatGobacit/Ciccus.

Red Andaluza de la Nueva Cultura del Agua (s/f). «Mapa colaborativo de los conflictos del agua en Andalucía». Recuperado de https://redandaluzaagua.org/ mapa/

Red Andaluza de la Nueva Cultura del Agua (2017). «¿Quiénes somos?» Recuperado de http://redandaluzaagua.org/presentacion/

Risler, Julia y Ares, Pablo (2015). «Talleres de mapeo. Recursos lúdicos y visuales para la construcción de conocimiento colectivo». Ecología Política, 48.

Ruiz-Mallén, I. (2020). «Co-production and resilient cities to climate change». En Nared, J. y Bole, D. (eds.), Participatory Research and Planning in Practice (pp. 1-12). Reino Unido: Springer Open.

Sorrentino, M., Sicilia, M. y Howlett, M. (2018). «Understanding co-production as a new public governance tool». Policy and Society, 37(3), pp. 277-293. DOI: http://doi.org/10.1080/14494035.2018.1521676.

Subirats, J. (2011). Otra sociedad, ¿otra política? De «no nos representan» a la democracia de lo común. Barcelona: Icaria. 


\section{Leandro del Moral | Belén Pedregal | Cesare laconi | Antonio Figueroa}

María Mancilla | Violeta Cabello

Subirats, J. (2016). «El papel de la ciudadanía en la coproducción de políticas urbanas. Entre la innovación social y la nueva institucionalidad». En Álvarez,

L. (ed.), Ciudadanía y nuevos actores en grandes ciudades (pp. 45-70). México: UNAM/UAM/Juan Pablos.

Swyngedouw, E. (2019). «La economía política y la ecología política del ciclo hidro-social». En Castro, J.E., Kohan, G., Poma, A. y Ruggerio, C. (eds.), Territorialidades del agua. Conocimiento y acción para construir el futuro que queremos (pp. 48-57). Buenos Aires: WaterlatGobacit/Ciccus.

Swyngedouw, E. (2011). «Interrogating post-democratization: reclaiming egalitarian political spaces». Political Geography, 30(7), pp. 370-380.

Tàbara, D. e Ilhan, A. (2008). «Culture as trigger for sustainability transition in the water domain: the case of the Spanish water policy and the Ebro river basin». Regional Environmental Change, 8(2), pp. 59-71.

Temper, L., Del Bene, D. y Martínez-Alier, J. (2015). «Mapping the frontiers and front lines of global environmental justice: the EJAtlas». Journal of Political Ecology, 22, pp. 255-278.

Van der Hel, S. (2016). «New science for global sustainability? The institutionalisation of knowledge co-production in future Earth». Environmental Science \& Policy, 61, pp. 165-175. DOI: http://doi.org/10.1016/j.envsci.2016.03.012

Van Kerkhoff, L.E. y Lebel L. (2015). «Coproductive capacities: rethinking science-governance relations in a diverse world». Ecology and Society, 20(1), p. 14. DOI: https://doi.org/10.5751/ES-07188-200114 